\section{RSP}

http://www.rsp.fsp.usp.br/
Revista de Saúde Pública

\title{
Recommendations of physical activity and rest in a Colombian prenatal control program
}

\author{
Myriam Ruiz-Rodríguez' iD, Yuri Sánchez-Martínez' \\ iD, Paula Camila Ramírez-Muñoz" iD, \\ Diana Marina Camargo-Lemos" iD \\ I Universidad Industrial de Santander. Departamento de Salud Pública. Bucaramanga, Colombia \\ " Universidad Industrial de Santander. Escuela de Fisioterapia. Bucaramanga, Colombia
}

\section{ABSTRACT}

OBJECTIVE: To determine the frequency of the registry of physical activity and rest recommendations made to pregnant women and to explore their associated factors in a prenatal care program of primary care public institutions in Bucaramanga, Colombia.

METHODS: An observational study was conducted. The sampling frame consisted of the medical records of the pregnant women who attended at least one prenatal care program between January 1 and December 31, $2012(\mathrm{n}=2.932)$, in 21 primary care health centers. We analyzed sociodemographic variables, prenatal and clinical antecedents, and information related to health personnel and the organization of health centers as possible factors associated with the recommendations of physical activity and rest recorded in the clinical history. Logistic regression models were applied to explore associations with $\alpha=0.10$.

RESULTS: There was a frequency of $26.1 \%$ of PA recommendations and $3.6 \%$ of rest recommendation on record, issued by nutrition (97.3\%) and medical (86.7\%) professionals, respectively. The factors associated with the registration of physical activity recommendations were: being nulliparous pregnant $(\mathrm{OR}=1.7$ ), attending more than four Prenatal Care Attention Programs $(\mathrm{OR}=2.2)$, having high or medium obstetric risk in the first prenatal care program $(\mathrm{OR}=0.6)$, and being attended in the western $(\mathrm{OR}=0.5)$ and eastern $(\mathrm{OR}=0.2)$ administrative areas health centers.

CONCLUSIONS: The low frequency of physical activity recommendations found in the records makes it necessary to reinforce the management strategies of health centers and strengthen the monitoring and accompaniment to comply with the care protocols. In addition, it is necessary to train health teams on the benefits of physical activity and their proper prescription, considering the multiple benefits derived from their practice on the maternal-fetal health.

DESCRIPTORS: Pregnant Women. Exercise.Rest. Prenatal Care. 


\section{INTRODUCTION}

Prenatal care is defined as the care provided, in general by health professionals, to all pregnant women to ensure the best health conditions for the woman and her fetus ${ }^{1}$. As in pregnancy women feel motivated to improve their habits and lifestyles to benefit their health and their child's, the recommendations that health personnel provide to pregnant women are important ${ }^{2}$. Advice on healthy habits includes proper diet, physical activity (PA) practice and the appropriate weight gain during pregnancy ${ }^{3}$.

Scientific evidence shows that the practice of PA in pregnant women is associated with a lower probability of developing hypertensive alterations in pregnancy ${ }^{4}$, diabetes mellitus ${ }^{5}$, preterm delivery ${ }^{6}$ and cesarean section 7 . In addition, it contributes to a better control of weight gain during pregnancy ${ }^{6}$ and brings benefits to maternal mental health, decreasing the possibility of anxiety ${ }^{8}$ and depressive symptoms ${ }^{9}$. Therefore, it is necessary for health professionals to recommend and educate pregnant women about the regular practice of PA.

Despite the importance of PA for maternal-fetal health, a large part of the health staff does not offer recommendations on its practice and, in addition, its duration, frequency and intensity is not clear ${ }^{10}$. On the other hand, absolute rest is recommended, even in situations not clinically indicated ${ }^{11}$, despite the harmful effects it may have on the health of the mother-child binomial ${ }^{12}$.

Additional factors of cultural nature also have a negative influence on the practice of PA during pregnancy, among them the fear of harming the fetus or inducing an abortion, beliefs that have been transmitted and perpetuated by family and friends $\mathrm{s}^{10,13}$. These factors, added to the lack of clarity by health personnel when offering recommendations, represent one of the most significant barriers to PA practice during pregnancy ${ }^{14}$.

Studies through maternal interviews indicate that between $39 \%$ and $55 \%$ of women do not receive education about their health care $^{15,16}$. Other studies have focused on establishing the frequency of PA and whether this practice is based on a recommendation from health personnel ${ }^{14,17}$. However, few studies have explored how much prenatal care providers agreed with PA recommendations ${ }^{18}$. Few studies have quantified the record in the clinical history of PA and rest recommendations by health personnel during prenatal care; Yeoh, in a study from Malasya, found that this record existed in only $3.3 \%$ of medical records ${ }^{16}$.

On the other hand, in the literature reviewed only two studies reported the frequency with which pregnant women discussed with the health personnel in the prenatal control programs (PNCP) about rest. White et al. found that $76.9 \%$ of 1265 pregnant women interviewed by telephone reported having talked to health personnel about the need for rest during pregnancy ${ }^{15}$. On the other hand, Yeoh et al. ${ }^{16}$, in their study based on the review of medical records, only registered the average number of times that pregnant women received recommendations, finding that this was a regular practice in PNCP, with an average of 2.7, against the average of 0.03 times of physical activity recommendations.

In the literature reviewed in Colombia, only two studies evaluated compliance with the activities carried out in the PCPN ${ }^{19,20}$; Arias et al. ${ }^{19}$ found that more than $50 \%$ of the pregnant women interviewed received PA recommendations from the nursing staff; and Álvarez et al..$^{20}$, although they evaluated that the health team agreed with the PNCP protocol by reviewing medical records and interviewing users, they did not measure how much they agreed with the provision of PA and rest recommendations from the clinical record. To know the frequency of PA and rest recommendations registry will contribute to the decision making by the directors of the PNCP aimed at reinforcing the PA counseling, identifying the areas that require interventions; therefore, this work aimed to determine the frequency of the PA and rest recommendations registry in the pregnant women's medical records and to explore their associated factors in a primary care level PNCP in Bucaramanga, Colombia. 


\section{METHODS}

\section{Context of the Research}

The study was carried out in Bucaramanga, capital of the department of Santander (Colombia). This city is nationally recognized because since 2009 it has been implementing strategies with a Primary Health Care (PHC) approach that transcend those stipulated by the General System of Social Security in Health.

The municipal health authority is the Secretary of Health and Environment, which - through the Social Enterprise of the State Institute of Health of Bucaramanga (ESE-ISABU) - provides primary health care services for low-income population served in 24 urban health centers (HC), two rural mobile HCs, an urban extramural team, a Maternal and Child Intermediate Health Unit (UIMIST) and a Local Hospital (LH).

Bucaramanga is divided into 17 communes, defined as administrative subdivisions made up of neighborhoods, settlements, urbanizations and other sectors with a floating population ${ }^{21}$. The HCs offer their services in 12 of the 17 communes of the city, which corresponds to $45 \%$ of the total population, characterized by being poor, with health insurance coverage via State subsidies. For their administration and supervision, the HCs are organized by administrative zones: north, east, west and south, with the northern zone being the most socioeconomically depressed; each area has an assigned coordinator, who manages the programs that the HCs provide.

ESE-ISABU offers low-risk PNCP in HCs. For their operation, they have health professionals. All pregnant women are evaluated by medical, nursing and dentistry professionals; attention for nutrition and psychology is offered according to the particular needs of each pregnant woman and based on the referral made by medical and nursing professionals. When women in their first control or in a subsequent control are classified as high risk, they are referred to the UIMIST or the LH; if the problem is solved, they are sent back to the respective HC so that they can continue their control.

All HCs follow the guidelines of Resolution 412 of the year 2000, specified in the National Technical Standard for the Detection of Pregnancy Disorders ${ }^{22}$, and those of the low-risk prenatal care protocol, typical of the ESE-ISABU, which contains activities complementary to those proposed by the national standard. Both Resolution 412 and the ESE-ISABU protocol recommend carrying out individual education for pregnant women on PA; however, neither of these two documents specifies the content of these recommendations in terms of frequency, intensity and duration of the PA.

It is necessary to establish the differences between a recommendation, a prescription and an education about human behavior in a control program. The first is related to counseling on the importance of performing PA; the second specifically contains the type of PA and the domains of duration, frequency and intensity, in order to improve health condition; finally, the third is related to a bidirectional and interactive process of behavioral change, from learning and making decisions aimed at improving health and well-being, in which the health personnel must ensure the understanding of information about which one is being educated ${ }^{23}$.

\section{Source of Information for Analysis}

A secondary analysis of the information collected in the project called "Technical efficiency of health promotion and disease prevention programs for women in the public primary care network of Bucaramanga, Colombia" was carried out. The methodology of this project is widely described in Ruiz-Rodríguez et al. ${ }^{24}$

The sampling frame consisted of the medical records of all pregnant women who attended at least one Prenatal Control (PNC) between January 1 and December 31, 2012 ( $\mathrm{n}=2,932)$ 
in $21 \mathrm{HCs}$ of public first-level care, belonging to the ESE-ISABU. A random sample was selected between 16 and 60 clinical records in each $\mathrm{HC}$, proportional to the total of pregnant women attended in each HC during 2012.

The research team, with the support of nutrition, nursing and medical professionals with experience in PNCP, generated a checklist to review the medical records. The checklist was adjusted after a pilot test with all the items included in the clinical record defined by the ESE-ISABU for the PNCP. The staff that supported the research team in the preparation of the checklist and pilot test took care of its completion, after training and standardization of procedures. In the case of PA and rest, the information of each PNC was carefully reviewed in the diagnostic and care plan sections.

\section{Variables}

The presence of the record of PA and rest recommendation by medical, nursing and nutrition professionals are the dependent variables; these variables were dichotomized as yes or no.

The independent variables of the pregnant women, registered in the clinical record and included for the analysis, were:

a. Sociodemographic: age (years old completed), education (last school year approved), economically remunerated occupation (yes, no), marital status, according to cohabitation with partner (yes, no) and socioeconomic stratum (1, 2 and $\geq 3,1$ being the lowest).

b. Anthropometric measurements: pre-gestational body mass index (BMI) categorized as low weight, normal weight and excess weight, taking as reference the cut-off points of the World Health Organization (WHO).

c. Background: nulliparity (yes, no), presence of at least one obstetric history such as diabetes, pregnancy-induced hypertension, preeclampsia, eclampsia or kidney disease in previous pregnancies (yes, no).

d. Related to the PNCP: gestational age at the beginning of the PNCP (trimester), high or medium obstetric risk at the first PNC (yes, no), number of PNC performed (1-4 or > 4), diagnosis of at least one health condition such as anemia, threatened abortion, placenta previa or intrauterine growth restriction, according to laboratory results and ultrasound in at least one NPC (yes, no).

e. Administrative service area (north, west, east and south).

\section{Analysis}

A descriptive analysis of the pregnant women characteristics was carried out through measures of central tendency and dispersion for the continuous variables and frequency tables for the categorical variables. The bivariate analysis was carried out among the dependent variables, with each of the continuous independent variables using the Mann-Whitney U test, and in the case of the categorical ones the chi-squared test was applied.

To estimate the factors associated with PA recommendation, a crude and adjusted analysis was made between the independent variables with the PA recommendation record, applying a logistic regression model following the Greenland ${ }^{25}$ recommendations to estimate the odds ratio and 95\%CI; this model was adjusted for pre-pregnancy BMI, nulliparity, number of PNC performed, diagnosis of high or medium obstetric risk in the first PNC and administrative care area.

Given the low frequency of rest recommendations, it was not possible to elaborate a multivariate model, so only associations of bivariate analysis are shown. All the analyzes were carried out in Stata 14.1 with a level of significance $\alpha=0.10$, given the exploratory nature of this work.

The study was endorsed by the ethics committee of the Bucaramanga Health Institute. 


\section{RESULTS}

\section{General Description}

We reviewed the medical records of 838 pregnant women between 13 and 46 years old who attended at least one PNC between January 1 and December 31, 2012 at 21 HCs of Bucaramanga. The medians of age and schooling years were 22 years $(\mathrm{RIC}=19-27)$ and nine years (RIC $=6-11$ ) respectively; $60.5 \%$ of pregnant women were nulliparous, $59.5 \%$ started PNC in the first gestational trimester and $56.6 \%$ were classified as high or medium obstetric risk during the first PNC (Table 1).

\section{Recommendations of PA}

PA recommendation was found in $26.1 \%$ of pregnant women's medical records; however, its type, time, frequency or duration was not specified. This registry was made mainly by

Table 1. Sociodemographic, anthropometric characteristics and antecedents registered in the clinical records of pregnant women attending prenatal control programs in Bucaramanga, Colombia. $(\mathrm{n}=838)$

\begin{tabular}{|c|c|c|}
\hline Characteristics & $\mathbf{n}$ & $\%$ \\
\hline \multicolumn{3}{|c|}{ Sociodemographic } \\
\hline Age (years old) Median (RIC) & 22 & $19-27$ \\
\hline Education (years) Median (RIC) & 9 & $6-11$ \\
\hline Paid occupation & 270 & 32.3 \\
\hline Live with partner & 637 & 77.6 \\
\hline \multicolumn{3}{|l|}{ Socioeconomic stratum } \\
\hline 1 & 329 & 39.7 \\
\hline 2 & 309 & 37.3 \\
\hline$>3$ & 190 & 22.9 \\
\hline \multicolumn{3}{|c|}{ Anthropometry } \\
\hline \multicolumn{3}{|l|}{ BMI (first PNC) } \\
\hline Under Weight & 65 & 8.0 \\
\hline Normal weight & 476 & 58.3 \\
\hline Overweight & 275 & 33.7 \\
\hline \multicolumn{3}{|c|}{ Background } \\
\hline Nulliparous & 505 & 60.5 \\
\hline Obstetric history ${ }^{\mathrm{a}}$ & 353 & 42.2 \\
\hline \multicolumn{3}{|c|}{ Related to the PNCP } \\
\hline \multicolumn{3}{|l|}{ Gestational trimester (first PNC) } \\
\hline 1 & 465 & 59.5 \\
\hline 2 & 227 & 29.0 \\
\hline 3 & 90 & 11.5 \\
\hline Obstetric risk (first PNC) ${ }^{\mathrm{b}}$ & 427 & 56.6 \\
\hline More than 4 PNCs made & 457 & 54.5 \\
\hline Diagnostics during $\mathrm{PNC}^{\mathrm{a}, \mathrm{c}}$ & 128 & 15.3 \\
\hline \multicolumn{3}{|c|}{ Administrative area of the care center } \\
\hline North & 250 & 29.8 \\
\hline West & 229 & 27.3 \\
\hline East & 166 & 19.8 \\
\hline South & 193 & 23.0 \\
\hline \multicolumn{3}{|c|}{$\begin{array}{l}\text { RIC: interquartile range; BMI: body mass index; PNC: prenatal control; PNCP: Prenatal Control Program } \\
\text { a Includes diagnosis of diabetes, pregnancy-induced hypertension, preeclampsia, eclampsia or kidney disease in } \\
\text { previous pregnancies. } \\
\text { b Includes high and medium obstetric risk. } \\
\text { c Includes diagnoses of anemia, threatened abortion, placenta previa or intrauterine growth restriction in at least } \\
\text { one prenatal check-up. }\end{array}$} \\
\hline
\end{tabular}


nutrition professionals (97.3\%), compared with medical (2.7\%); it is emphasized that the nursing staff did not make PA recommendations.

In the bivariate analysis of factors associated with PA recommendation record (Table 2), it was found that pregnant women belonging to the lowest socioeconomic stratum, known in Colombia as stratum 1, with normal pregestational weight, nulliparous, in the first gestational trimester at the time of the first PNC, attended more than four PNCs, that were not diagnosed as high or medium obstetric risk in the first PNC, and that performed PNCs in northern zone institutions were more likely to register a PA recommendation in their medical record (Table 2).

Table 2. Sociodemographic, anthropometric, clinical characteristics and personal history of pregnant women, according to the registry of physical activity and rest recommendation in the clinical record.

\begin{tabular}{|c|c|c|c|c|c|c|c|c|c|c|}
\hline \multirow{3}{*}{ Characteristic } & \multicolumn{4}{|c|}{ Registry of PA recommendations } & \multirow{3}{*}{ p } & \multicolumn{4}{|c|}{ Registry of rest recommendations } & \multirow{3}{*}{$\mathbf{p}$} \\
\hline & \multicolumn{2}{|c|}{ Yes $(n=219)$} & \multicolumn{2}{|c|}{ No $(n=619)$} & & \multicolumn{2}{|c|}{ Yes $(n=30)$} & \multicolumn{2}{|c|}{ No $(n=808)$} & \\
\hline & $\mathbf{n}$ & $\%$ & n & $\%$ & & $\mathrm{n}$ & $\%$ & $n$ & $\%$ & \\
\hline \multicolumn{11}{|c|}{ Characteristics } \\
\hline Age (years old) Median (RIC) & 22 & $18-26$ & 23 & $19-27$ & 0.037 & 25 & $21-27$ & 22 & $19-27$ & 0.051 \\
\hline $\begin{array}{l}\text { Education (years) Median } \\
\text { (RIC) }\end{array}$ & 10 & $6-11$ & 9 & $6-11$ & 0.507 & 7.5 & $5-11$ & 9 & $6-11$ & 0.160 \\
\hline Paid occupation & 66 & 30.1 & 204 & 33.1 & 0.426 & 8 & 26.7 & 262 & 32.5 & 0.502 \\
\hline Live with partner & 171 & 79.5 & 466 & 76.9 & 0.426 & 26 & 92.9 & 611 & 77.0 & 0.049 \\
\hline \multicolumn{11}{|l|}{ Socioeconomic stratum } \\
\hline 1 & 97 & 44.7 & 232 & 38.0 & & 14 & 46.7 & 315 & 39.5 & \\
\hline 2 & 88 & 40.5 & 221 & 36.2 & 0.004 & 14 & 46.7 & 295 & 37.0 & 0.095 \\
\hline$>3$ & 32 & 14.7 & 158 & 25.9 & & 2 & 6.7 & 188 & 23.6 & \\
\hline \multicolumn{11}{|c|}{ Anthropometry } \\
\hline
\end{tabular}

BMI (first PNC)

$\begin{array}{lcccccccccc}\text { Under Weight } & 14 & 6.4 & 51 & 8.5 & & 2 & 6.7 & 63 & 8.0 & \\ \text { Normal weight } & 144 & 66.4 & 332 & 55.4 & 0.020 & 19 & 63.3 & 457 & 58.1 & 0.850 \\ \text { Overweight } & 59 & 27.2 & 216 & 36.1 & & 9 & 30.0 & 266 & 33.8 & \\ \text { Medical History } & & & & & & & & & & \\ \text { Nulliparous } & 148 & 67.6 & 357 & 57.9 & 0.012 & 10 & 33.3 & 495 & 61.5 & 0.002 \\ \text { Obstetric history }^{\text {ab }} & 89 & 40.6 & 264 & 42.8 & 0.580 & 13 & 43.3 & 340 & 42.2 & 0.900\end{array}$

Related to the PNCP

Gestational trimester (first

PNC)

\begin{tabular}{|c|c|c|c|c|c|c|c|c|c|c|}
\hline 1 & 135 & 66.2 & 330 & 57.1 & 0.032 & 25 & 86.2 & 440 & 58.4 & 0.009 \\
\hline 2 & 54 & 26.5 & 173 & 29.9 & & 4 & 13.8 & 223 & 29.6 & \\
\hline 3 & 15 & 7.3 & 75 & 13.0 & & 0 & 0 & 90 & 11.9 & \\
\hline Obstetric risk (first $\mathrm{PNC})^{\mathrm{b}}$ & 78 & 40.4 & 349 & 62.1 & $<0.001$ & 15 & 62.5 & 412 & 56.4 & 0.551 \\
\hline More than 4 PNCs made & 157 & 71.7 & 300 & 48.5 & $<0.001$ & 24 & 80.0 & 433 & 53.6 & 0.004 \\
\hline Diagnostics during $\mathrm{PNC}^{\mathrm{c}}$ & 33 & 15.1 & 95 & 15.3 & 0.921 & 3 & 10.0 & 125 & 15.5 & 0.413 \\
\hline \multicolumn{11}{|c|}{ Administrative area of the care center } \\
\hline North & 100 & 45.7 & 150 & 24.2 & \multirow{4}{*}{$<0.001$} & 11 & 36.7 & 239 & 29.6 & \multirow{4}{*}{0.491} \\
\hline West & 51 & 23.3 & 178 & 28.8 & & 6 & 20.0 & 223 & 27.6 & \\
\hline East & 13 & 5.9 & 153 & 24,7 & & 4 & 13.3 & 162 & 20.0 & \\
\hline South & 55 & 25.1 & 138 & 22.3 & & 9 & 30.0 & 184 & 22.8 & \\
\hline
\end{tabular}

RIC: interquartile range; BMI: body mass index; PNC: prenatal control; PNCP: Prenatal Control Program

The differences in the PA and rest recommendations according to the independent variables were estimated by the Mann-Whitney $U$ test for age and education and with the chi-square test for the other variables.

a Includes diagnosis of diabetes, pregnancy-induced hypertension, preeclampsia, eclampsia or kidney disease in previous pregnancies.

${ }^{\mathrm{b}}$ Includes high and medium obstetric risk.

c Includes diagnoses of anemia, threatened abortion, placenta previa or intrauterine growth restriction in at least one prenatal check-up. 
Table 3. Factors associated with the registration of physical activity recommendations to pregnant women attending prenatal care programs in Bucaramanga (Linktest $p=0.266$ ).

\begin{tabular}{|c|c|c|c|c|c|c|}
\hline \multirow{2}{*}{ Characteristic } & \multicolumn{3}{|c|}{ Not Adjusted } & \multicolumn{3}{|c|}{ Adjusted } \\
\hline & OR & $95 \% \mathrm{Cl}$ & $\mathbf{p}$ & OR & $95 \% \mathrm{Cl}$ & $\mathbf{p}$ \\
\hline \multicolumn{7}{|l|}{ BMI (first PNC) } \\
\hline Under Weight & 0.6 & $0.3-1.2$ & 0.15 & 0.6 & $0.3-1.2$ & 0.138 \\
\hline Normal weight & Ref. & & & Ref. & & \\
\hline Overweight & 0.6 & $0.4-0.9$ & 0.009 & 0.7 & $0.5-1.1$ & 0.103 \\
\hline \multicolumn{7}{|l|}{ Nulliparous } \\
\hline No & Ref. & & & Ref. & & \\
\hline Yes & 1.5 & $1.1-2.1$ & 0.013 & 1.7 & $1.1-2.4$ & 0.007 \\
\hline \multicolumn{7}{|c|}{ Obstetric risk (first PNC) } \\
\hline No & Ref. & & & Ref. & & \\
\hline Yes & 0.4 & $0.3-0.6$ & $<0.001$ & 0.6 & $0.4-0.9$ & 0.010 \\
\hline \multicolumn{7}{|l|}{ PNC number } \\
\hline $1-4$ & Ref. & & & Ref. & & \\
\hline$>4$ & 2.7 & $1.9-3.7$ & $<0.001$ & 2.2 & $1.5-3.2$ & $<0.001$ \\
\hline \multicolumn{7}{|c|}{$\begin{array}{l}\text { Administrative area of the care } \\
\text { center }\end{array}$} \\
\hline North & Ref. & & & Ref. & & \\
\hline West & 0.4 & $0.3-0.6$ & $<0.001$ & 0.5 & $0.3-0.8$ & 0.008 \\
\hline East & 0.1 & $0.1-0.2$ & $<0.001$ & 0.2 & $0.1-0.3$ & $<0.001$ \\
\hline South & 0.6 & $0.4-0.9$ & 0.012 & 0.8 & $0.5-1.3$ & 0.459 \\
\hline
\end{tabular}

BMI: body mass index; PNC: prenatal control.

The factors analyzed in the multivariate model showed positive and statistically significant associations for nulliparous pregnant women (OR $=1.795 \% \mathrm{CI} 1.1-2.4)$ and for those who attended more than four PNC (OR $=2.295 \% \mathrm{CI} 1.5-3.2)$; in contrast, negative and statistically significant associations were found between the PA recommendations and pregnant women with high or medium obstetric risk in the first PNC (OR $=0.695 \% \mathrm{CI} 0.4-0.9)$ and those that were attended in the $\mathrm{HC}$ of the west $(\mathrm{OR}=0.595 \% \mathrm{CI} 0.3-0.8)$ and east $(\mathrm{OR}=0.2$ 95\%CI 0.1-0.3) administrative areas (Table 3).

\section{Recommendations of Rest}

Only 3.6\% of PNCP users registered rest recommendations in the clinical record, with physicians prescribing it more frequently (86.7\%), comparing with nurses (13.3\%). Additionally, it was evidenced that pregnant women who lived with a partner, were multiparous, in the first gestational trimester at the time of the first PNC and attending later to more than four PNCs were more frequently registered with rest recommendations $(p<0.05)($ Table 2$)$.

\section{DISCUSSION}

The objective of this secondary analysis was to establish the frequency and possible factors associated with the registry of PA and rest recommendations in the clinical record of PNCP pregnant women in a Colombian city. Its importance lies in the fact that it is the first time that data are presented from the pregnant women's medical records, unlike other studies, which were based on interviews with women ${ }^{19,20}$. Although the information from clinical records has limitations, these are less than those that are derived from studies from surveys with long-term reminders, given their potential information bias.

Our results show that $26.1 \%$ of pregnant women had a record of PA recommendations in PNCP; this frequency is greater than $3.3 \%$ reported by Yeoh et al. ${ }^{16}$; the differences can be explained by the type of record used in each study. 
In the multivariate analysis, no sociodemographic characteristics of the pregnant women were associated with the registration of PA recommendations. On the other hand, nulliparity, the obstetric risk at the beginning of the PNC, the PNC number and the administrative area were associated. Having children was negatively associated with registering the PA recommendation during the PNC consultation. These findings coincide with that reported by Santo et al. ${ }^{17}$, who found that a high parity was significantly associated with PA recommendations by health personnel. To the date, a negative association between equality and PA practice during pregnancy has been documented, attributing this situation to the lack of time of some pregnant women, because they must take care of their children at home ${ }^{26}$.

In the sample analyzed, it was found that pregnant women who attended between one and four PNCs were rarely recommended on PA, compared with those who attended more than four PNCs; This may be because those who attended more than four PNCs were closer to the health personnel and received more detailed follow-up of their pregnancy, facilitating the counseling of the pregnant women in different aspects, including PA; however, in the literature reviewed there was no evidence to support this finding.

Our results showed a negative association between high or medium obstetric risk during the first PNC and the record of PA recommendations in the clinical record; this situation may be due to the abstention of health personnel to recommend the PA practice in this group of pregnant women for fear of harming the mother's or child's health ${ }^{13}$.

Additionally, we found statistically significant associations between the administrative area to which the $\mathrm{HC}$ belonged and the registry of PA recommendation; this probably shows differences in the administration of the $\mathrm{HC}$ in each zone and not in the conglomerate of socioeconomic strata that conform them, which is evidenced in the bivariate analysis results, considering that if it represented the socioeconomic stratum of the geographic zones, a significant effect of this variable would have also been found.

It has been found that during PNC consultations other aspects related to the health of pregnant women are prioritized and PA is relegated to the background ${ }^{19,27}$. Additionally, some authors have reported that one of the main barriers in prescribing PA is the lack of information by health professionals about international recommendations for safe PA practice ${ }^{14,27}$. In this sense, a qualitative study in pregnant Latinas residing in the United States showed that the participants expressed a certain degree of confusion by the health personnel when offering the PA counseling ${ }^{10}$. Contrary to what was observed in the PA prescription during pregnancy, the American College of Obstetricians and Gynecologists (ACOG) has warned about the high prescription of rest during pregnancy, even without being really indicated, despite its harmful effects on maternal-fetal health ${ }^{11,28}$.

Because of the importance of the role played by health personnel in maternal-fetal wellbeing, our findings pose challenges for the local health system. On the one hand, the need to reinforce $\mathrm{HC}$ management strategies and the strengthening of monitoring and accompaniment in compliance with care protocols; on the other hand, the need to train their health teams regarding the prescription of PA for pregnant women, so that they are aware that PA and rest are individual education interventions of the PNC consultation ${ }^{15,17,29}$. The fact that no detailed records on the PA recommendations were found in the medical records indicates that there is a lack of knowledge on how to make the prescription of PA for pregnant women. This situation also raises the need to formulate specific guides on pregnant PA in the framework of the PNCP. Likewise, the results of this study suggest the need for higher education institutions to include in their curricula PA and rest recommendations as an aspect of comprehensive care for pregnant women within the framework of the maternal and perinatal Ruta Integral de Atención en Salud (RIAS) that will begin to be implemented this year. 
Contrary to the aforementioned studies, our results showed that only $3.6 \%$ of pregnant women were recommended for rest. These differences can be explained by a possible underreporting of these recommendations by PNC professionals.

One of the main limitations of this study and, in particular, of the secondary analyzes derived from clinical histories, is that there is a potential underreporting of information, which would affect the frequencies of record detected and the potential associations to be evaluated. However, the random selection of the clinical histories analyzed during the year of analysis, as well as the sampling frame, makes it possible to generalize the findings.

On the other hand, more studies documenting the figures and factors associated with PA and rest recommendation in PNCP are needed to facilitate discussion and comparisons, in order to propose new strategies in Latin American countries to overcome the difficulties registered here.

\section{CONCLUSIONS}

In summary, a low frequency was found in the registry of PA recommendations and an even lower registry of rest recommendations during prenatal care in a socioeconomic vulnerable population. These findings suggest that being the PNCP a scenario with an enormous potential to provide counseling and recommendations on healthy practices, the users of the PNCP of the study did not have the opportunity to receive information or advice on practices regarding PA and rest, which could deepen even more their social vulnerability level. On the other hand, the preponderant role of nutrition professionals in the registry of recommendations, comparing with those of medical and nursing areas, is highlighted and also poses challenges in terms of training on PA and rest recommendation.

The results of this study are also a baseline for proposing strategies that increase both the quantity and quality of PA and rest recommendations, which will contribute to achieve health outcomes in maternal and perinatal RIAS within the framework of the new Comprehensive Health Care Policy (PAIS) promulgated in 2016.

\section{REFERENCES}

1. Abalos E, Chamillard M, Diaz V, Tuncalp O, Gülmezoglu AM. Antenatal care for healthy pregnant women: a mapping of interventions from existing guidelines to inform the development of new WHO guidance on antenatal care. BJOG. 2016;123(4):519-28. https://doi.org/10.1111/1471-0528.13820

2. Kominiarek MA, Peaceman AM. Gestational weight gain. Am J Obstet Gynecol. 2017(6);642-51. https://doi.org/10.1016/j.ajog.2017.05.040

3. World Health Organization. WHO recommendation on antenatal care for positive pregnancy experience. Geneva: WHO; 2016 [cited 2018 Mar 25]. Available from: http://apps.who.int/iris/ bitstream/10665/250796/1/9789241549912-eng.pdf

4. Di Mascio D, Magro-Malosso ER, Saccone G, Marhefka GD, Berghella V. Exercise during pregnancy in normal-weight women and risk of preterm birth: a systematic review and meta-analysis of randomized controlled trials. Am J Obstet Gynecol. 2016;215(5):561-71. https://doi.org/10.1016/j.ajog.2016.06.014

5. Zhang C, Solomon CG, Manson JE, Hu FB. A prospective study of pregravid physical activity and sedentary behaviors in relation to the risk for gestational diabetes mellitus. Arch Intern Med. 2006;166(5):543-8.https://doi.org/10.1001/archinte.166.5.543

6. Silva SG, Ricardo LI, Evenson KR, Hallal PC. Leisure-time physical activity in pregnancy and maternal-child health: a systematic review and meta-analysis of randomized controlled trials and cohort studies. Sports Med. 2017;47(2):295-317.https://doi.org/10.1007/s40279-016-0565-2

7. Dumith SC, Domingues MR, Mendoza-Sassi RA, Cesar JA. Atividade física durante a gestação e associação com indicadores de saúde materno-infantil. Rev Saude Publica. 2012;46(2):327-33. https://doi.org/10.1590/S0034-89102012005000012 
8. Takahasi EHM, Alves MTSSB, Alves GS, Silva AAM, Batista RFL, Simões VMF, et al. Mental health and physical inactivity during pregnancy: a cross-sectional study nested in the BRISA cohort study. Cad Saude Publica. 2013;29(8):1583-94. https://doi.org/10.1590/0102-311X00115112

9. Haas JS, Jackson RA, Fuentes-Afflick E, Stewart AL, Dean ML, Brawarsky P, et al. Changes in the health status of women during and after pregnancy. J Gen Intern Med. 2005;20(1):45-51. https://doi.org/10.1111/j.1525-1497.2004.40097.x

10. Lindsay AC, Wallington SF, Greaney ML, Tavares Machado MM, Andrade GP. Patient-provider communication and counseling about gestational weight gain and physical activity: a qualitative study of the perceptions and experiences of Latinas pregnant with their first child. Int J Environ Res Public Health. 2017;14(11):1412-24. https://doi.org/10.3390/ijerph14111412

11. American College of Obstetricians and Gynecologists. Physical activity and exercise during pregnancy and the postpartum period committee opinion. Comm Opin. 2015;(650):268-73.

12. Nayak M, Peinhaupt M, Heinemann A, Eekhoff MEW, Mechelen W, Desoye G, et al. Sedentary behavior in obese pregnant women is associated with inflammatory markers and lipid profile but not with glucose metabolism. Cytokine. 2016;88:91-8. https://doi.org/10.1016/j.cyto.2016.08.031

13. Evenson KR, Moos MK, Carrier K, Siega-Riz AM, et al. Perceived barriers to physical activity among pregnant women. Matern Child Health J. 2009;13(3):364-75. https://doi.org/10.1007/s10995-008-0359-8

14. Whitaker KM, Wilcox S, Liu J, Blair SN, Pate RR. Provider advice and women's intentions to meet weight gain, physical activity, and nutrition guidelines during pregnancy. Matern Child Health J. 2016;20(11):2309-17. https://doi.org/10.1007/s10995-016-2054-5

15. White DE, Fraser-Lee NJ, Tough S, Newburn-Cook CV. The content of prenatal care and its relationship to preterm birth in Alberta, Canada. Health Care Women Int. 2006;27(9):777-92. https://doi.org/10.1080/07399330600880335

16. Yeoh PL, Hornetz K, Shauki NIA, Dahlui M. Assessing the extent of adherence to the recommended antenatal care content in Malaysia: room for improvement. PLoS One. 2015;10(8):e0135301. https://doi.org/10.1371/journal.pone.0135301

17. Santo EC, Forbes PW, Oken E, Belfort MB. Determinants of physical activity frequency and provider advice during pregnancy. BMC Pregnancy Childbirth. 2017;17(1):286-96. https://doi.org/10.1186/s12884-017-1460-z

18. Schmidt SM, Chari R, Davenport MH. Exercise during pregnancy: current recommendations by Canadian maternity health care providers. J Obstet Gynaecol Can. 2016;38(2):177-8. https://doi.org/10.1016/j.jogc.2015.12.012

19. Arias Charry J, Bernal Arias K, Giraldo Carvajal DC, Gonzalez Echeverri EV, Rivera Mejía PT. Calidad del control prenatal realizado por profesionales de enfermería en una IPS publica, Manizales 2007. Hacia Promoc Salud. 2008;13(1):131-42.

20. Alvarez J, Caballero F, Castaño J, Escobar D, Franco A, Gómez P, et al. Cumplimiento de las actividades de promoción y prevención en el control prenatal en una entida de $1^{\circ}$ nivel, Manizales, años 2005-2007. ArchMed. 2010;10(1):19-30.

21. Alcaldía de Bucaramanga (CO). División Político Urbana. Bucaramanga (CO); 2018 [cited 2018 Mar 25]. Available from: http://www.bucaramanga.gov.co/el-mapa/division-politico-urbana/

22. Ministerio de Salud (CO), Dirección General de Promoción y Prevención. Norma técnica para la detección temprana de problemas en el embarazo. Bogotá; 2000 [cited 2018 Mar 25]. Available from: https://www.minsalud.gov.co/sites/rid/Lists/BibliotecaDigital/RIDE/VS/PP/normatecnica-para-la-deteccion-temprana-embarazo.pdf

23. Nolan ML. Information giving and education in pregnancy: a review of qualitative studies. J Perinat Educ. 2009;18(4):21-30. https://doi.org/10.1624/105812409X474681

24. Ruiz-Rodriguez M, Rodriguez-Villamizar LA, Heredia-Pi I. Technical efficiency of women's health prevention programs in Bucaramanga, Colombia: a four-stage analysis. BMC Health Serv Res. 2016;16(1):576-88. https://doi.org/10.1186/s12913-016-1837-0

25. Greenland S. Modeling and variable selection in epidemiologic analysis. Am J Public Health. 1989;79(3):340-9.https://doi.org/10.2105/AJPH.79.3.340

26. Domingues MR, Barros AJD. Leisure-time physical activity during pregnancy in the 2004 Pelotas Birth Cohort Study. Rev Saude Publica. 2007;41(2):173-80. https://doi.org/10.1590/S0034-89102007000200002 
27. Nascimento SL, Surita FG, Godoy AC, Kasawara KT, Morais SS. Physical activity patterns and factors related to exercise during pregnancy: a cross sectional study. PLoS One. 2015;10(6):e0128953. https://doi.org/10.1371/journal.pone.0128953

28. Artal R, O'Toole M. Guidelines of the American College of Obstetricians and Gynecologists for exercise during pregnancy and the postpartum period. Br J Sports Med. 2003;37(1):6-12. https://doi.org/10.1136/bjsm.37.1.6

29. Coll CVN, Domingues MR, Gonc H, Bertoldi AD. Perceived barriers to leisure-time physical activity during pregnancy: a literature review of quantitative and qualitative evidence. J Sci Med Sport. 2017;20():17-25. https://doi.org/10.1016/j.jsams.2016.06.007

Funding: Universidad Industrial de Santander (UIS), Secretary of Health and Environment of Bucaramanga and Empresa Social del Estado Instituto de Salud de Bucaramanga (ESE-ISABU - Cooperation Agreement 11-2012).

Authors' Contribution: Design and planning of the study: MRR. Analysis and interpretation of data: MRR, YSM, PCRM, DMCL. Elaboration and critical revision of the manuscript, approval of the final version: MRR, YSM, PCRM, DMCL. Responsabilidad pública por el contenido del artículo: MRR, YSM, PCRM, DMCL.

Conflict of Interest: The authors declare no conflict of interests. 